\title{
RECHERCHES SUR LES SILICONES
}

PAR

\section{O. BOUDOUARD}

On désigne sous le nom de silicones les composés obtenus par l'action de l'acide chlorhydrique sur certains siliciures métalliques et présentant la propriété d'être décomposés lentement par l'action de l'eau avec dégagement d’hydrogène.

Buff et Wöhler ont les premiers obtenu ces corps, soit par l'action de l'eau sur les composés chlorés et iodés du silicium, soit par l'action de l'acide chlorhydrique sur le siliciure de calcium (1). Geuther a étudié le produit résultant de l'attaque du siliciure de magnésium par l'acide chlorhydrique (2). Wöhler a décrit sous le nom particulier de silicone un composé de couleur jaune orangé formé de lamelles transparentes, obtenu par l'action de l'acide chlorhydrique sur le siliciure de calcium, et sous le nom de leucone le produit provenant de l'action de la lumière et de l'eau sur la silicone (3). Ces différents auteurs ont considéré ces divers composés comme des hydrates siliciques correspondant à des oxydes inférieurs à $\mathrm{Si}^{2}$; les formules qu'ils leur ont attribuées étaient d'ailleurs très complexes.

MM. Friedel et Ladenburg, au contraire, en étudiant l'action de l'eau sur le silicichloroforme et sur le sesquiiodure de silicium, ont décrit deux composés ternaires partaitement définis que, par analogie avec des composés semblables du carbone, ils ont appelés anhydride siliciformique et hydrate silicioxalique (4); leurs formules respectives sont $\mathrm{Si}^{2} \mathrm{O}^{3} \mathrm{H}^{2}$ et $\mathrm{Si}^{2} \mathrm{O}^{4} \mathrm{H}^{2}$. MM. Troost et Hautefeuille, dans leur étude sur les composés halogénés du silicium ont obtenu les mêmes corps par action de l'eau sur le protochlorure et le sesquichlorure de silicium (ّ̋).

Le tableau ci-dessous permet de comparer les résultats obtenus par ces divers savants; les nombres indiquent les compositions centésimales ( $v$. le tableau page suivante).

Les silicones se présentent à l'état amorphe et sont diversement colorées suivant leur origine. Leurs propriétés chimiques sont sensiblement les mêmes ; insolubilité dans les acides, sauf l'acide fluorhydrique ; décomposition avec dégagement d'hydrogène par l'eau, les alcalis, l'ammoniaque, les carbonates alcalins ; réduction des sels métalliques en présence des alcalis.

Lorsqu'on attaque une fonte par l'acide chlorhydrique, on obtient un résidu

(1) Lieb. Annalen, t. CIV, p. 94 et 374.

(2) J. prakt Ch., t. XCV, p. 424.

(3) Lieb. Annalen, t. CXXVII, p. 257 ; Ann. Ch. Phys. (3) t. LXIX, p. 224.

(4) Comptes-rendus, t. LXIV, p. 359.

(5) Bull. Soc. chim. (2), t. VII, p. 322 ; Lieb. Annalen, t. CXLIII, p. 118 ; t. CCIII, p. 250 ; Gatrвkmann, Berichte, t. XXII, p. 192;Ann. Ch. Phys. (5), t. VII, p. 463. 


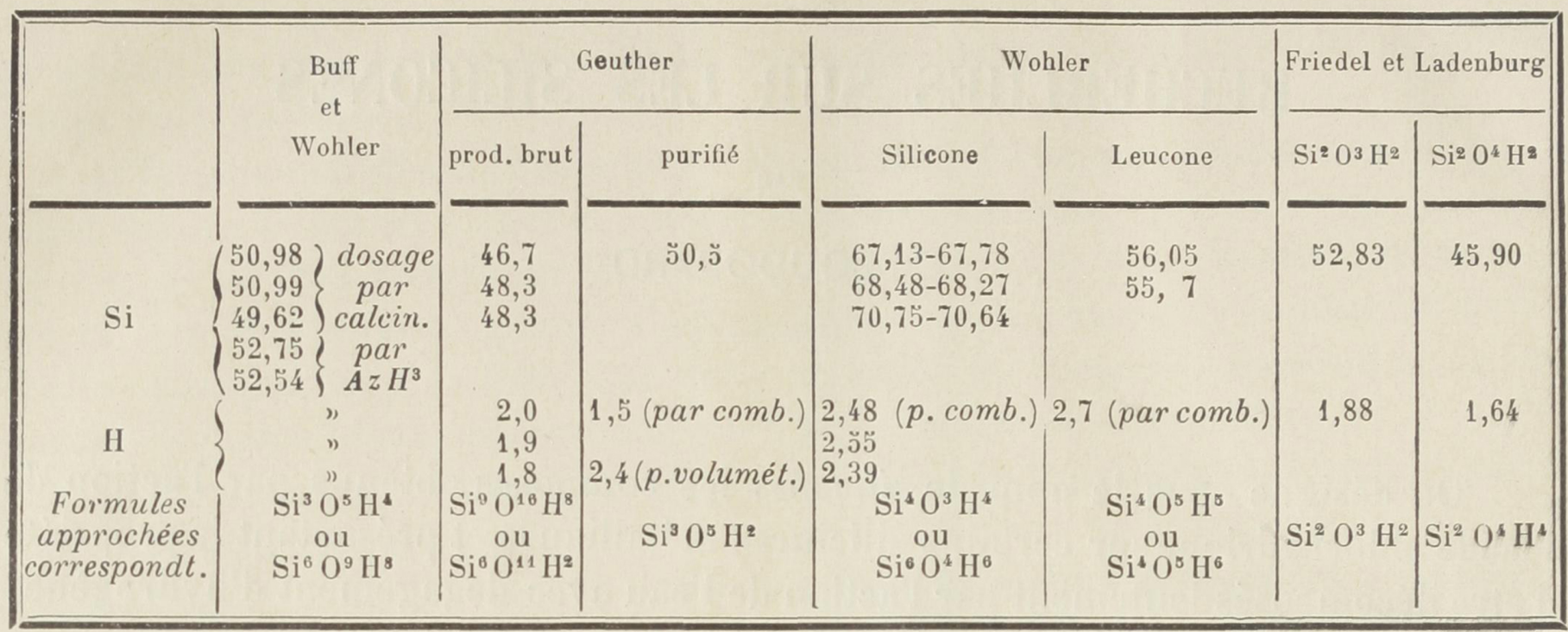

de couleur gris-noirâtre qui contient une certaine quantité de silicone : les alcalis en effet donnent un abondant dégagement d'hydrogène. Je me suis alors proposé d'étudier les différentes silicones données par des aciers au silicium que je dois à l'obligeance de M. Guillet; la composition de ces aciers était la suivante :

\begin{tabular}{|c|c|c|c|c|c|}
\hline Désignation & C & $\mathrm{Si}$ & S & $P$ & $\mathrm{Mn}$ \\
\hline $\begin{array}{l}\text { G } 2 \\
\text { G } 3 \\
\text { G } 5\end{array}$ & $\begin{array}{l}0,209 \\
0,177 \\
0,277\end{array}$ & $\begin{array}{l}0,932 \\
1,60 \\
5,12\end{array}$ & $\begin{array}{l}0,020 \\
0,012 \\
0,009\end{array}$ & $\begin{array}{l}0,024 \\
0,032 \\
0,03 \text { ' }\end{array}$ & $\begin{array}{r}\text { traces } \\
0275 \\
0,380\end{array}$ \\
\hline
\end{tabular}

Les silicones sont obtenues de la façon suivante : on attaque l'acier par l'acide chlorhydrique à chaud jusqu'à complète dissolution du fer; on recueille sur filtre le résidu insoluble, on lave complètement à l'eau froide aussi rapidement que possible, on sèche entre des feuilles de papier filtre et on met le produit obtenu sur l'acide sulfurique, ou mieux dans le vide en présence d'acide sulfurique. Au bout de quelques jours, on a une silicone se présentant sous la forme d'une poudre amorphe; la couleur est assez variable, allant du blanc à un gris plus ou moins foncé.

Les matières ainsi préparées retiennent toujours une certaine quantité d'eau hygroscopique qui ne disparaît pas complètement même à $150^{\circ}$. Elles ne renferment pas tout le silicium contenu dans les aciers traités : la quantité de métalloïde retrouvé varie généralement entre 90 et 100 0/0, mais elle est quelquefois inférieure (elle est descendue à $570 / 0$ dans la silicone obtenue à froid avec le métal G.5้). Si tout le silicium s'était trouvé à l'état combiné dans les silicones, il eût alors été possible d'éviter l'évaporation à sec, toujours si fastidieuse lors des dosages de silice; le tableau suivant donne les résultats d'essais entrepris pour déterminer l'ordre de grandeur des erreurs possibles en supprimant l'évaporation à sec. Ces essais ont été ainsi conduits : l’acier est attaqué par l'acide chlorhydrique chaud; on filtre et on lave la silicone; le liquide est évaporé à sec à $110^{\circ}$, repris par l'eau acide, et on récupère ainsi une certaine quantité de silice; cette opération est répétée une seconde fois. Les chitfres du tableau (p. suiv.) donnent les quantités pour 100 de silicium retrouvé par rapport au silicium total.

Pour analyser les silicones obtenues, j’ai employé la méthode décrite par 
Friedel et Ladenburg consistant à transformer la silicone en silice par action de l'ammoniaque étendue, évaporation à sec et calcination, d'une part; à mesurer le volume d'hydrogène dégagé par l'action de la potasse, d'autre part. Concur-

\begin{tabular}{|c|c|c|}
\hline & $1^{\text {ro }}$ évaporation à sec & 2 e évaporation à sec \\
\hline $\begin{array}{cc}\text { G } & 1 \\
\text { G } & 2 \\
\text { G } & 3 \\
\text { G } & 5\end{array}$ & $\begin{array}{r}14,7 \\
12,2 \\
10,5 \\
1,2\end{array}$ & $\begin{array}{l}2,1 \\
0,7 \\
0,3\end{array}$ \\
\hline
\end{tabular}

remment, j'ai fait des analyses par combustion, ce qui me donnait en plus les faibles quantités de carbone se trouvant mélangé à la silicone.

Les résultats analytiques sont consignés dans le tableau ci-dessous. Aux analyses des silicones obtenues par attaque des aciers cités plus haut, j'ai ajouté celles relatives à un anhydride siliciformique impur provenant de la préparation du tétrachlorure de silicium, à une silicone obtenue par attaque à froid de l'acier G 5; à des silicones obtenues par attaque d'un siliciure de fer préparé par l'aluminothermie et contenant $100 / 0$ de silicium. L'attaque de ce siliciure faite à chaud par l'acide chlorhydrique a été très longue, et j'ai recueilli en deux fois le produit obtenu pour voir si une action prolongée du réactif d’attaque ne modifiait pas la composition du produit final :

\begin{tabular}{|c|c|c|c|c|c|c|c|c|}
\hline Désignation & $\begin{array}{c}\text { Eau } \\
\grave{a} \\
150^{\circ}\end{array}$ & Garbone & $\begin{array}{c}\mathrm{H} \\
\text { corrigé } \\
\text { (combustion) }\end{array}$ & $\begin{array}{r}\text { col } \\
\text { (par } P\end{array}$ & tigé & $\begin{array}{c}\mathrm{Si} \\
\text { corrigé } \\
\text { (combustion) }\end{array}$ & $\begin{array}{c}\mathbf{S i} \\
\text { corrigé } \\
(p . \text { ammoniaque })\end{array}$ & $\begin{array}{c}\mathrm{Si} \\
\text { moyenne }\end{array}$ \\
\hline $\begin{array}{c}\text { Anh. silicif. impur } \\
\text { G } 2 \\
\text { G } 3 \\
\text { G } 5 \\
\text { G } 5 \\
\text { (faite à froid) } \\
\text { Aluminothermie } \\
\text { 1er dépôt } \\
2^{\text {e dépôt }}\end{array}$ & $\begin{array}{l}0,88 \\
1,5 \\
0,18 \\
2,58 \\
20,6\end{array}$ & $\begin{array}{c}\text { " } \\
2,26 \\
2,51 \\
3,23 \\
1,02\end{array}$ & $\begin{array}{l}1, \pi 6 \\
3,03 \\
1,94 \\
2,93 \\
3,24\end{array}$ & $\begin{array}{l}2,62 \\
1,69 \\
1,44 \\
2,22 \\
2,82 \\
\\
0,61 \\
0,62\end{array}$ & $\begin{array}{l}1,31 \\
0,85 \\
0,72 \\
1,11 \\
1,41 \\
\\
\\
0,305 \\
0,31\end{array}$ & $\begin{array}{c}47,6 \\
47,6 \\
46,9 \\
50,98 \\
50,0-50,7-50,4\end{array}$ & $\begin{array}{c}50,4-48,1-47,6 \\
46,4-47,2 \\
46,6-46,9 \\
52,1-53,4-50,3 \\
50,7\end{array}$ & $\begin{array}{l}48,4 \\
47,1 \\
46,8 \\
51,5 \\
50,3\end{array}$ \\
\hline
\end{tabular}

Pour discuter ces résultats, je rappellerai la composition centésimale de composés ternaires décrits par Friedel et Ladenburg :

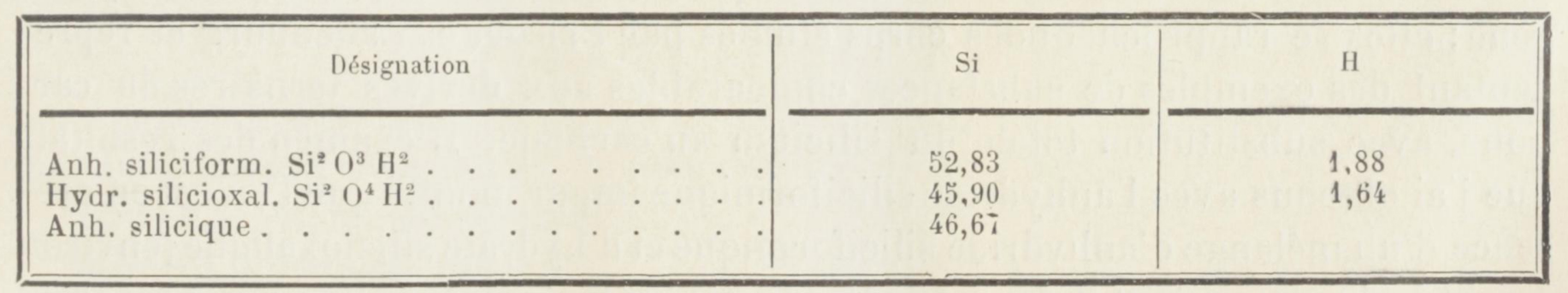

L'action des alcalis sur ces corps produit un dégagement d'hydrogène; comme le montrent les formules de réaction, avec l'anhydride siliciformique, le volume d'hydrogène dégagé est double de celui existant réellement en combinaison; avec l’hydrate silicioxalique, au contraire, il lui est égal.

$$
\begin{aligned}
& \mathrm{Si}^{2} O^{3} \mathrm{H}^{2}+4 \mathrm{KOH}=2 \mathrm{Si} O^{3} \mathrm{~K}^{2}+\mathrm{H}^{2} \mathrm{O}+2 \mathrm{H}^{2} \\
& \mathrm{Si}^{2} \mathrm{O}^{4} \mathrm{H}^{2}+4 \mathrm{KOH}=2 \mathrm{Si} \mathrm{O}^{3} \mathrm{~K}^{2}+2 \mathrm{H}^{2} \mathrm{O}+\mathrm{H}^{2}
\end{aligned}
$$


C'est pour cette raison que dans la colonne donnant les quantités d'hydrogène dégagé, j’ai mis deux nombres pour chacun des essais : le premier correspond à la quantité d'hydrogène observée expérimentalement, le second est égal à la moitié du précédent.

Les silicones sont des composés qu'il est difficile de préparer à l'état anhydre sous l'action de la chaleur; à partir de $200^{\circ}$, il y a déjà transformation partielle, et à l'ébullition du soufre, il y a altération profonde du produit initial.

Silicone G 5. - Prise d'essai : 1,7846

après 2 heures de chauffage à $100^{\circ}: 1,7712$, soit $0,750 / 0$

$150^{\circ}: 1,7712$, -

après 4 heures de chauffage à $185^{\circ}: 1,7707$, soit $0,780 / 0$

après 2 heures de chauffage à $225^{\circ}: 1,8006$

L'analyse ci-dessous se rapporte à la même silicone chauffée à $445^{\circ}$ pendant une heure :

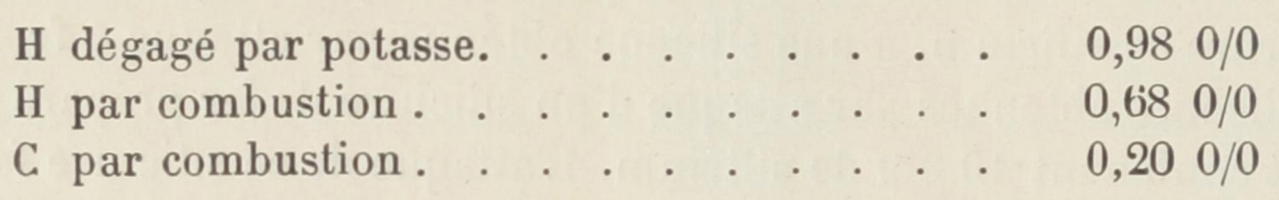

L'augmentation de poids de la silicone chauffée de $150^{\circ}$ à $445^{\circ}\left(0^{\mathrm{gr}}, 0211\right.$, la prise d'essai étant $0 \mathrm{gr}^{\mathrm{r}}, 4810$ ) montre qu'il y a eu transformation partielle en silice; du car bone a déjà été brûlé, et alors que dans les autres essais, la quantité d'hydrogène trouvée par combustion est notablement plus grande que celle trouvée par action des alcalis, le fait inverse est observé pour la silicone préalablement chauffée à $44 \mathrm{O}^{\circ}$.

Etant données les difficultés d'obtention de produits absolument secs, à moins d'un séjour extrêmement prolongé dans le vide sulfurique, les dosages volumétriques d'hydrogène devront donc seuls être envisagés si l'on veut déterminer la composition des silicones. Quant à la faible teneur en hydrogène des silicones obtenues avec le siliciure de fer fait par aluminothermie, elle s'explique facilement en admettant que l'action très prolongée à chaud de l'acide chlorhydrique doit décomposer le produit obtenu initialement: la silicone $\mathbf{G} 5$ faite à froid contient plus d'hydrogène que la silicone $G$ s faite à chaud.

En résumé, les silicones sont des composés ternaires renfermant de l'hydrogène; elles se rapprochent des corps étudiés par Friedel et Ladenburg et représentant des exemples de substances comparables aux dérivés ternaires du carbone, avec substitution totale du silicium au carbone. L'examen des résultats que j'ai obtenus avec l'anhydride siliciformique impur montre que l'on est en présence d'un mélange d'anhydride siliciformique et d'hydrate silicioxalique (environ 1 partie du premier et 2 du second); de même les silicones que j’ai préparées peuvent être considér ées comme des mélanges de même nature en proportions variables. Il est certain qu'on n'arrive pas à des composés parfaitement définis par suite de la difficulté d'attaque des siliciures par l'acide chlorhydrique et du contact très prolongé entre la silicone, l'acide et l'eau qui en est la conséquence immédiate. 